\title{
INVESTIGACIONES
}

\section{El consejo escolar del Instituto Nacional (José Miguel Carrera) desde una perspectiva de deliberación curricular}

\author{
The school council of Instituto Nacional (José Miguel Carrera) \\ from a perspective of curricular deliberation \\ O conselho escolar do Instituto Nacional José Miguel Carrera \\ desde uma perspectiva de deliberação curricular
}

Claudio Lozano $G$.

Pontificia Universidad Católica de Chile

Telf.: 66953116. Correo electrónico: calozano@uc.cl

\begin{abstract}
RESUMEN
La constitución de consejos escolares, órgano que por ley posee carácter informativo, propositivo y consultivo, es obligatoria desde 2004. Sin embargo, y en un contexto de aguda y prolongada presión social demandando transformaciones en educación, en 2013 el Consejo municipal de la comuna de Santiago resuelve otorgarle carácter resolutivo a los consejos escolares del municipio en tres de las seis materias que establece la ley, las cuales pueden considerarse de interés curricular. En esta indagación se analiza al consejo escolar del Instituto Nacional José Miguel Carrera (CEIN) y su dinámica en la construcción de curriculum con el propósito de develar si se constituye en un espacio democrático de diálogo y deliberación curricular. La presencia de consejeros con intereses, prácticas y jerarquías distintas o el establecimiento del CEIN como una política 'desde arriba', permiten discutir acerca de las condiciones de deliberación en el marco de este órgano.
\end{abstract}

Palabras clave: consejo escolar, deliberación, curriculum, democracia.

\section{ABSTRACT}

The constitution of a school council, authority that legally has an informative, propositional and consultative role, is compulsory since 2004. However, in a context of strong social pressure regarding educational changes, in 2013 the city council of Santiago commune determined to grant an operative character to the school councils of the municipality in three out of six points established by the law as of curricular importance. This inquiry leads to the analysis of the school council from Instituto Nacional José Miguel Carrera (CEIN) and its dynamics in the setting of the curriculum with the purpose of revealing if it is constituted in a democratic setting of dialogue and curricular deliberation. The presence of councilors with different interests, practices and hierarchies or the establishment of CEIN as a policy "from above" allow to discuss about the conditions of deliberation in the framework of this organization.

Key words: school council, deliberation, curriculum, democracy. 


\section{INTRODUCCIÓN}

El presente artículo corresponde a una indagación sobre el consejo escolar del Instituto Nacional José Miguel Carrera ${ }^{1}$, enfocada a analizar su dinámica en la construcción de curriculum. La política de consejos escolares, establecida mediante la ley 19.979 de 2004, es promovida como "una invitación a hacer de la escuela y el liceo un espacio de formación ciudadana, que acoja la diversidad e integre las opiniones y necesidades de sus miembros, enriqueciendo así su proyecto educativo" (DEM, 2014). A pesar de que existen pocos antecedentes que ilustren acerca del progreso de esta política ${ }^{2}$, en abril de 2013 el Consejo municipal de la comuna de Santiago resuelve otorgarle facultad resolutiva a los consejos escolares de los establecimientos dependientes del Departamento de Educación Municipal (DEM), sosteniendo su alcaldesa que "la única manera de reconstruir las confianzas es escuchando, poniéndose de acuerdo, que es lo que corresponde en una instancia que es educativa"3. En tanto, y desde los referentes que sustentan esta indagación, las materias respecto de las cuales los consejos escolares de la comuna poseen carácter resolutivo (1. Proyecto Educativo Institucional, 2. Programación anual y actividades extracurriculares y 3. Revisión, modificaciones y aprobación del reglamento interno del establecimiento) pueden considerarse de interés curricular.

Sin embargo, y lo más relevante, ¿existen en los establecimientos condiciones que permitan acoger la diversidad e integrar las opiniones y necesidades de los miembros?, ¿cómo actúan y con cuáles propósitos lo hacen los consejeros y los estamentos en un proceso de acuerdo en el marco del consejo escolar?, ¿sus acuerdos son siempre racionales, legítimos o democráticos? Esta indagación se centra más precisamente en visualizar al CEIN desde una perspectiva de deliberación curricular, ello comprendiendo que la política de consejos escolares se identifica, a lo menos declarativamente, con el establecimiento de acuerdos, que en ciertos casos deben alcanzarse en materias de interés curricular. En tal sentido, es preciso considerar que en el marco del CEIN se relacionan agentes con trayectorias, funciones, jerarquías, propósitos e intereses en muchos casos distintos, lo cual alerta sobre la eventualidad de que los acuerdos que allí se alcancen sigan fundándose en relaciones asimétricas de poder o se establezcan en el marco de un dispositivo todavía posible de caracterizar como autoritario, ilegítimo o antidemocrático. Por ello el propósito general de esta indagación es develar si el CEIN se constituye como un espacio de deliberación curricular.

La noción de deliberación se recoge de Habermas (1999), quién junto con aportar en su conceptualización, propone condiciones y procedimientos para su realización, mientras que la noción de curriculum se recoge de una tradición crítica, especialmente de los aportes de Giroux (1983), Grundy (1998), Da Silva (1999) y, más recientemente, de Ferrada (2001, 2008). En términos metodológicos, se ha compendiado y seleccionado, entre otros documentos, las actas publicadas del CEIN durante el periodo julio de 2013 a abril de 2014 y se ha realizado entrevistas semiestructuradas a tres de sus consejeros, información que ha sido sometida luego a un diseño de análisis crítico del discurso (ACD) desde un enfoque histórico (Wodak

\footnotetext{
En adelante CEIN.

Destaca a este respecto la tesis de Magíster de C. Drago (2008), "Los consejos escolares como espacios de participación de las comunidades educativas", disponible en www.opech.cl

Artículo de prensa recuperado el día 31 de octubre de 2014 del sitio http://www.lanacion.cl/consejos-escolares-seranresolutivos-en-todos-los-colegios-de-santiago/noticias/2013-04-26/163804.html
} 
2003). Los resultados se presentarán atendiendo a tres dimensiones de análisis: 1. Contexto histórico / político, 2. Intereses o ideologías curriculares y 3. Deliberación curricular.

Por último, se considera relevante esta indagación en un contexto de amplio debate sobre políticas en educación, en el cual se demanda de parte de ciertos agentes, otrora excluidos, mayores grados de participación y no meramente testimonial. Visualizar no solo los consejos escolares, sino incluso las prácticas en aula desde una perspectiva de deliberación curricular, permite avanzar en una reconceptualización y evolución práctica de la escuela como un espacio de 'formación ciudadana', inspirado y sustentado en nociones y procedimientos más democráticos. La política de los consejos escolares resolutivos constituye una oportunidad en este sentido, pues establece, a lo menos a priori, la existencia de un órgano colegiado, pluriestamental y de cogobierno institucional en las materias que son de su competencia, capaz de responder a las necesidades de mayor integración y participación de los miembros de las comunidades. Sin embargo, le acecha a su vez un riesgo implícito, y es que bajo la apariencia de mayor participación se podría eventualmente estar legitimando formas de presión, cooptación, burocratismo, asimetrías de poder, etc., lo cual, ciertamente, y a nuestro entender, pervertiría el espíritu declarado de esta política.

\section{ANTECEDENTES}

El problema de develar si el CEIN se constituye como un espacio de deliberación de curriculum, debe ser planteado, en primer término y en general, como un problema político, ello en virtud de que el curriculum que eventualmente se dialoga y/o delibera en el CEIN depende precisamente de la constitución y desarrollo de ese espacio, proceso posible de identificar en todo momento con intereses, voluntades y/o prácticas de índole política. En tal sentido es que el presente marco indaga, en primer término, en el modelo habermasiano de democracia deliberativa, intentando encontrar allí los criterios o condiciones que permitan, primero, considerar al CEIN como un espacio de esta naturaleza.

Luego se cree preciso relacionar la práctica deliberativa con los intereses curriculares involucrados y con los discursos que de ella emergen, ello con la intención de considerar o descartar al CEIN, en segundo término, como un espacio de deliberación curricular. Para ello se ha indagado en: 1. Una concepción crítica del curriculum, 2. La aplicación curricular de la teoría de los intereses constitutivos de saberes, aporte a la teoría curricular realizado por Grundy (1998) y 3. La especificación curricular de ciertas orientaciones de la teoría crítica, denominada perspectiva crítica-comunicativa del curriculum, formuladas, entre otros, por Ferrada (2001 y 2008).

Por último, interesa discutir en este marco acerca de las facultades (consultivas y/o resolutivas), los grados de formalidad, racionalidad y legitimidad de este eventual espacio de deliberación curricular, para lo cual se acude a la normativa vigente (ley 19.979, ley 20.370 y ordenanza 294), vinculándola con las nociones de racionalidad y legitimidad desarrolladas por el propio Habermas (1999).

\subsection{ACERCA DE LA NOCIÓN DE DELIBERACIÓN}

La noción de participación democrática de los miembros de las comunidades educativas en el diálogo y deliberación en torno al curriculum puede considerarse como una 
especificación (extensión, aplicación) al ámbito educacional-curricular de la teoría y modelo de democracia deliberativa propuesto por Habermas (1999). Un problema fundamental que dicho modelo pretende abordar es el de las pretensiones de validez y/o legitimidad de las decisiones que se adoptan en el terreno público, cuestionables en ausencia de un proceso previo de implicación de los agentes involucrados y de deliberación de su parte.

Desde esta perspectiva, y en tanto ámbito de interés de los miembros de las comunidades educativas, el curriculum es susceptible de ser sometido a deliberación, proceso que en términos de Habermas “(...) hace referencia a una cierta actitud propicia a la cooperación social, a saber, a esa disposición abierta a ser persuadido mediante razones relativas a las demandas de los otros como a las propias" (Habermas, 1999: 237). La noción de democracia deliberativa, señala Cohen: “(...) está enraizada en el ideal intuitivo de una asociación democrática en la que la justificación de los términos y condiciones de la asociación tienen lugar a través de la argumentación y del razonamiento público entre ciudadanos iguales" (Cit. en Habermas, 1998: 381).

Joshua Cohen, citado por Habermas (1998), propone ciertos principios, condiciones o procedimientos (formas) que deben observarse durante el proceso deliberativo, so perjuicio de incurrir en decisiones ilegítimas o irracionales, condiciones posibles de resumir y esquematizar de la siguiente manera:

a. La deliberación democrática implica un intercambio regulado de informaciones y razones.

b. Estas razones deben ser inclusivas y públicas.

c. Deben estar exentas de coerciones externas e internas.

d. Las deliberaciones se orientan a alcanzar un acuerdo racionalmente motivado y pueden proseguirse ilimitadamente o retomarse en cualquier momento, y aun así, a las deliberaciones políticas se les pone un término mediante el acuerdo mayoritario.

e. Las deliberaciones se deben extender, en principio, a la totalidad de las materias.

f. Las deliberaciones políticas han de extenderse a la interpretación de las necesidades y al cambio de actitudes y preferencias prepolíticas (Cit. en Habermas, 1998: 382-383)4.

La información de los agentes, la construcción de opinión y voluntad, el pronunciamiento por parte de todos los agentes involucrados, el debate y construcción de consenso vinculante hasta un nuevo proceso deliberativo (Habermas, 1998), parecieran formas, habituales incluso, en contextos de escuelas democráticas (Apple y Beane, 2005).

\subsection{ACERCA DE LA NOCIÓN DE CURRICULUM}

Considerando la posibilidad de que el CEIN se constituya en un espacio de deliberación curricular, resulta luego necesario indagar en una noción de curriculum que sea coherente con la práctica deliberativa y que nos permita identificar cuáles materias sometidas a deliberación poseen relevancia curricular. Teniendo en cuenta la constitución y desarrollo del CEIN como un espacio político-institucional, una aproximación a la noción de curriculum nos insta a concebirlo como:

No obstante Habermas considera insuficientes las condiciones de Cohen sosteniendo que: "se echa en falta toda afirmación acerca de la relación entre el aspecto formal y el aspecto informal en la formación de la opinión y la voluntad, es decir, entre las deliberaciones orientadas a la decisión que vienen reguladas por procedimientos democráticos y los procesos informales de formación de la opinión en el espacio público-político" (Habermas 1998: 384). 
a. una manifestación de intereses políticos-culturales mediatizados por relaciones de poder, dimensión en la cual cabe citar a Da Silva, quien sostiene que: "Con las teorías críticas aprendemos que el currículo es, definitivamente, un espacio de poder" (Da Silva, 1999: 35), agregando que "las teorías críticas y pos-críticas del currículo están preocupadas con las conexiones entre saber, identidad y poder" (Da Silva, 1999: 6). Más adelante atribuye a Giroux la idea de la pedagogía y el currículo comprendidas como "política cultural, estrechamente ligados a relaciones sociales de poder y desigualdad" (Da Silva, 1999: 28).

b. que constituyen, por lo tanto, una construcción social de significados. Desde la perspectiva de Giroux, sintetizada por Da Silva, "el currículo involucra la construcción de significados y valores culturales (...) Es el lugar donde activamente se producen y crean significados sociales". Dicha idea es coherente con la planteada por Grundy, quién sostiene que: "el curriculum no es un concepto, sino una construcción cultural (...) no se trata de un concepto abstracto que tenga alguna existencia aparte de y antecedente a la experiencia humana" (Grundy, 1998: 19-20), noción posible de integrar con la planteada por Da Silva, quien sostiene que:

Después de las teorías críticas y pos-críticas resulta imposible pensar el currículo simplemente a través de conceptos técnicos como los de enseñanza y eficiencia o de categorías psicológicas como las de aprendizaje y desarrollo o también de imágenes estáticas como las de grado curricular y lista de contenidos (Da Silva, 1999: 34).

c. tales intereses se vinculan con intereses epistemológicos (constitutivos de saberes).

Cabe enunciar en esta dimensión que, según Grundy, dichos intereses “(...) configuran lo que consideramos que constituye el conocimiento y determinan las categorías mediante las que lo organizamos" (Grundy, 1998: 25).

d. dichos intereses políticos y epistemológicos, mediados por relaciones de poder, orientarán la construcción acerca del ¿qué conocer? y ¿por qué o para qué conocer?, orientándose especialmente al aprendizaje de los estudiantes. Cabe destacar en esta dimensión a Da Silva, quien sostiene que: "La cuestión central que sirve de plano de fondo para cualquier teoría del currículo es la de saber qué conocimiento debe ser enseñado", a lo cual agrega que "en las teorías del currículo, la pregunta ¿qué? nunca está separada de otra importante pregunta: ¿cómo deben ser las personas?, o mejor, ¿qué es lo que ellos y ellas deberán ser?". (Da Silva, 1999: 5). En este sentido, y de manera amplia, se entenderá que las materias de relevancia curricular tratadas en el marco del CEIN corresponden a todas aquellas que guarden relación con estas interrogantes.

Una importante especificación de las teorías de Habermas al campo educacionalcurricular es aquella que realiza Grundy (1998), quien sustentándose en la teoría de los intereses constitutivos de saberes, formulada por Habermas a comienzos de los 70, establece tres tipos de intereses epistemológicos que inspiran las distintas teorías y prácticas curriculares, a saber: 1 . un interés técnico: orientado al control de la naturaleza y la sociedad, vinculado con las ciencias empírico-analíticas; 2 . un interés práctico, orientado a la comprensión de la naturaleza y la sociedad, vinculado con las ciencias históricohermenéuticas y 3. un interés emancipador, orientado a la "potenciación (...) de individuos y grupos para tomar las riendas de sus propias vidas de manera autónoma y responsable" (Grundy, 1998: 38), vinculado con las ciencias críticas. 
Respecto del vínculo de este último interés con las ciencias críticas (teoría crítica), sostiene Habermas que el enfoque de las ciencias críticamente orientadas incluye el interés cognitivo emancipador (Habermas, 1982), idea que complementa Grundy planteando que, "el interés emancipador genera teorías críticas (...) teorías acerca de las personas y sobre la sociedad que explican cómo actúan la restricción y la deformación para inhibir la libertad" (Grundy, 1998: 39). Las nociones de diálogo democrático y deliberación de curriculum pueden vincularse con un interés emancipador en tanto, "compromete al estudiante no solo como receptor activo, en vez de pasivo, del saber, sino como creador activo del mismo junto con el profesor", al mismo tiempo que concibe a "(...) los estudiantes y el profesor ocupados de manera conjunta como participantes activos en la construcción del conocimiento" (Grundy, 1991: 142).

Coherente con lo anterior, se vincula una perspectiva crítica / comunicativa del curriculum, desde la cual se sostiene que las prácticas dialógicas (participación comunicativa) responden a los requerimientos de aprendizaje de los estudiantes en el contexto de las sociedades actuales, respuestas que los enfoques curriculares tradicionales (tecnológico, racionalista académico, cognitivo, etc.) no han sido capaces de ofrecer (Ferrada, 2001). En tanto especificación de la teoría de la acción comunicativa (Habermas, 1987) y desde una perspectiva crítica / comunicativa del curriculum, se plantea que la apertura de la participación comunicativa en la escuela se constituye en fuente de inclusión social, cultural y política (Ferrada, 2001). Desde este enfoque, las instancias de participación comunicativa:

\footnotetext{
(...) permiten protagonismo a los sujetos educativos, con lo cual se promueve la valoración intersubjetiva, al mismo tiempo que surgen nuevas relaciones de legitimidad y valoración de patrones normativos y conocimientos excluidos, omitidos, rechazados y prohibidos de la escuela, a la vez que somete a cuestionamiento tanto a éstos como a los tradicionalmente incluidos, aceptados y permitidos (Ferrada, 2001: 301-302).
}

\subsection{LA NORMATIVA VIGENTE Y LAS NOCIONES DE RACIONALIDAD Y LEGITIMIDAD}

La participación, a lo menos consultiva, de los miembros de las comunidades educativas en la toma de decisiones curriculares, particularmente en la construcción de los PEI, se encuentra además prevista en la legislación vigente. Desde 2004 la ley 19.979 establece la obligatoriedad de los establecimientos subvencionados de constituir consejos escolares, órgano que conforme al art. 15 de la ley 20.370 (LGE) tiene como propósito "estimular y canalizar la participación de la comunidad educativa en el proyecto educativo y en las demás áreas que estén dentro de la esfera de sus competencias". La propia ley 19.979 prevé un mínimo de materias que deben consultarse con el consejo escolar, pudiéndose, no obstante y por decisión del sostenedor, agregar materias y dotarlo de facultad resolutiva en alguna(s) de ella(s). Entre las materias que pueden considerarse de interés curricular y cuya consulta al consejo escolar la ley obliga se encuentran: 1. Proyecto Educativo Institucional (PEI), 2. Programación Anual y actividades extracurriculares, 3. Las metas del establecimiento y los proyectos de mejoramiento propuestos y 4. La elaboración y las modificaciones al reglamento interno del establecimiento.

La existencia jurídica de tal órgano supone un mínimo de resguardo y promoción de la participación de los estamentos al interior de los establecimientos, tal como lo expresa el DEM de la comuna de Santiago en su sitio, señalando que: 
Constituir el consejo escolar en cada uno de los establecimientos subvencionados del país (...) es una invitación a hacer de la escuela y el liceo un espacio de formación ciudadana, que acoja la diversidad e integre las opiniones y necesidades de sus miembros, enriqueciendo así su proyecto educativo (DEM, 2014).

Aunque cabe discutir si el carácter consultivo de los consejos escolares permite considerarlos como órganos democráticos, destaca a este respecto la ordenanza 294 del Consejo municipal de la comuna de Santiago (Abril, 2013) que otorga facultades resolutivas a los consejos escolares de los establecimientos municipales de la comuna en tres de las materias previstas en la ley 19.979, a saber: 1. Proyecto educativo institucional, 2. Programación anual y actividades extracurriculares y 3. Reglamento interno del establecimiento ${ }^{5}$.

Más allá de la existencia de normas que declaran promover con mayores o menores niveles de democracia la participación de los miembros de las comunidades educativas en la toma de decisiones curriculares, el problema de la ausencia de participación o de participación testimonial, reflejado en la no constitución de consejos escolares u otros espacios institucionalizados de diálogo y/o deliberación de curriculum, permite a los agentes involucrados cuestionar la legitimidad del curriculum. En cuanto a la noción de legitimidad, que en Habermas se encuentra estrechamente vinculada con la noción de racionalidad, sostiene el autor: "Todo depende, pues, de las condiciones de la comunicación y de los procedimientos que prestan su fuerza legitimadora a la formación institucionalizada de la opinión y de la voluntad común" (Habermas, 1999: 239). En cuanto a la vinculación con la noción de racionalidad, sostiene que:

El (...) modelo de democracia que yo quisiera proponer, se apoya precisamente en las condiciones de comunicación bajo las que el proceso político puede tener a su favor la presunción de generar resultados racionales, porque se efectúa en toda su extensión en el modo y estilo de la política deliberativa (Habermas, 1999: 239).

Luego agrega que: "La política deliberativa obtiene su fuerza legitimadora de la estructura discursiva de una formación de la opinión y la voluntad que solo puede cumplir su función socio integradora gracias a la expectativa de calidad racional de sus resultados" (Habermas, 1998: 381).

La legitimación del curriculum institucional, al relacionarse con procesos de deliberación, puede considerarse también como una forma de apropiación, lo cual nos permite vincular la legitimidad del curriculum con la significatividad que puede poseer para los agentes. La ausencia de espacios institucionalizados de diálogo y/o deliberación de curriculum puede relacionarse a su vez con múltiples factores: carencia de tradición o cultura deliberativa, desconocimiento o falta de interés de parte de los agentes involucrados, presencia de barreras autoritarias (dispositivos) que manifiesta o tácitamente los coartan, etc.

Considerando lo anterior, el objetivo general de esta indagación es develar si el CEIN se constituye como un espacio democrático de diálogo y/o deliberación de curriculum, mientras que los objetivos específicos se enumeran de la siguiente manera: a. Comprender el contexto

Más recientemente, a comienzos de septiembre de 2014, la Asociación chilena de municipalidades (ACHM) firma un acuerdo con representantes de la Coordinadora nacional de estudiantes secundarios (CONES) para dotar de carácter resolutivo a los consejos escolares de los establecimientos dependientes de los municipios asociados. 
-histórico y político- en el cual se desenvuelve el CEIN, b. Visibilizar las tensiones ideológicas y las relaciones de poder que se despliegan al interior del CEIN en torno a la construcción curricular y c. Analizar críticamente los discursos curriculares desplegados en el CEIN.

\section{METODOLOGÍA}

\subsection{TIPO DE INVESTIGACIÓN}

Dada la naturaleza del problema, la presente indagación se funda en un paradigma investigativo crítico, especificado en un análisis crítico del discurso (ACD) desde un enfoque histórico (Wodak, 2003). Es de tipo cualitativa, dado que su propósito es comprender el fenómeno de diálogo democrático y/o deliberación de curriculum en el CEIN de manera inductiva, atendiendo al contexto propio en el cual se desenvuelve (Albert, 2007). Se desarrollará además mediante la metodología de análisis de caso (Stake, 1998), puesto que se aspira al "entendimiento comprehensivo de dicha situación, el cual se obtiene a través de la descripción y análisis de la situación, la cual es tomada como un conjunto y dentro de su contexto" (Morra y Friedlander, 2001: 2). Dados los objetivos de la investigación, puede sostenerse que es un estudio de caso intrínseco (Stake, 1998) y de tipo descriptivo ilustrativo o de situación crítica (Morra y Friedlander, 2001). Por su parte, y en razón del estudio de una sola unidad de análisis (CEIN), puede caracterizarse como un estudio de caso único (Stake, 1998). En razón de los propósitos de la indagación, la muestra seleccionada será de tipo teórico intencional (Ruiz, 1996), abarcando el contexto (unidad de muestreo) antes referido. La muestra estará compuesta principalmente por el CEIN, cuya composición se detallará más adelante.

\subsection{RECOGIDA DE INFORMACIÓN}

La información que nutre la presente indagación está constituida principalmente por: 1. Actas del CEIN publicadas entre el periodo julio de 2013 a abril de 2014 y 2. Entrevistas semiestructuradas realizadas a tres de sus consejeros.

Respecto de las actas del CEIN se logró compendiar desde el sitio web institucional del

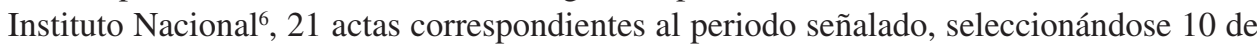
ellas $^{7}$. El criterio de selección responde principalmente a la búsqueda de transcripciones de diálogos o resoluciones acerca de materias de interés curricular sucedidas durante sesiones del CEIN. En las actas descartadas, las transcripciones con ese contenido son, por lo general, nulas o demasiado sucintas. El criterio para estimar el periodo de consideración de actas (julio de 2013 a abril de 2014) responde a que en julio de 2013 comienza a publicarse las actas del CEIN informado de su carácter resolutivo, mientras que en abril de 2014 se resuelve dar comienzo a una primera etapa de análisis ${ }^{8}$.

En relación al criterio de selección de los entrevistados, este consistió principalmente en asegurar que a lo menos tres estamentos involucrados de modo protagónico en la construcción

(www.institutonacional.cl)

Actas correspondientes a las siguientes fechas: 02/08/13, 27/08/13, 28/08/13, 05/09/13, 12/09/13, 29/11/13, 06/12/13, $07 / 12 / 13,08 / 12 / 13,15 / 04 / 14$

8 Las actas se citarán señalando el número de día, slash, número de mes, dos puntos y número de página (ej.: 02/08: 1) 
del curriculum fueran representados, mediante entrevista, en esta indagación ${ }^{9}$, para lo cual se consideró imprescindible obtener entrevista de parte de: un representante del cuerpo directivo, un representante del Consejo general de profesores (CGP) y un representante del Centro de alumnos del Instituto Nacional (CAIN). Cabe declarar que los representantes del Departamento de educación municipal (DEM), del Centro general de padres y apoderados (CEGPAIN) y de los asistentes del establecimiento fueron considerados prescindibles, ello a partir de sus nulas o reducidas intervenciones en materias de interés curricular durante sesiones del CEIN, criterio posible de contrastar con las transcripciones en actas.

El guion de entrevista semiestructurada se elaboró a partir de una matriz inicial en la cual se desglosan, a partir de los objetivos específicos, categorías conceptuales y descriptores que informan la elaboración de las preguntas (14), tal como lo ilustra el Cuadro 1:

Cuadro 1. Matriz de guion de entrevista semiestructurada

\begin{tabular}{|c|c|c|}
\hline $\begin{array}{l}\text { Objetivos } \\
\text { específicos }\end{array}$ & $\begin{array}{l}\text { Categorías } \\
\text { conceptuales }\end{array}$ & Descripción \\
\hline $\begin{array}{l}\text { Objetivo } \\
\text { Comprender el } \\
\text { contexto (histó- } \\
\text { rico y político) } \\
\text { en el cual se } \\
\text { desenvuelve el } \\
\text { Consejo escolar } \\
\text { del I.N. }\end{array}$ & $\begin{array}{l}\text { Contexto } \\
\text { nacional } \\
(2011 / 2013) \text {. }\end{array}$ & $\begin{array}{l}\text { El contexto nacional afecta al } 1 \text {. ¿Cómo cree usted que } \\
\text { contexto institucional. Los plan- el contexto nacional desde } \\
\text { teamientos y movilizaciones so- } 2011 \text { ha afectado la realidad } \\
\text { ciales (específicamente estudian- institucional? } \\
\text { tiles) en torno al conflicto educa- } 2 \text {. ¿Cómo considera usted } \\
\text { cional, presionan a la autoridad que ha influido el contexto } \\
\text { quien responde implementando nacional desde } 2011 \text { en la } \\
\text { políticas de reestructuración actual configuración (carác- } \\
\text { institucional (específicamente el ter resolutivo) del consejo } \\
\text { otorgamiento de carácter resolu- escolar? } \\
\text { tivo del Consejo Escolar). }\end{array}$ \\
\hline
\end{tabular}

El guion aplicado finalmente acoge los comentarios formales y de contenido señalados por dos expertos investigadores ${ }^{10}$, a quienes recurrimos como validadores del instrumento. Las entrevistas se realizaron durante el mes de julio de $2014^{11}$, previa aprobación, de parte de cada uno de los consejeros, de los términos de una carta de consentimiento informado, que incluye, entre otros aspectos, la autorización para obtener registro de audio. La transcripción (tabulación) de las entrevistas se realizó intentando no alterar el flujo discursivo de los entrevistados, exceptuando la inclusión de algunos elementos sintácticos. Se resguardó también no incluir en la transcripción aquellos pasajes que los entrevistados solicitaron expresamente ${ }^{12}$.

\footnotetext{
9 Cabe señalar que el número de consejeros presentes en sesión fluctúa entre un número de 8 y 13, estableciéndose en el reglamento del CEIN, aprobado en 2013, un número de 18.

10 Ambos con el grado de Magíster en Curriculum.

11 Específicamente los días 07/07/14 (Rector), 15/07/14 (representante del CGP) y el 17/07/14 (representante del CAIN).

12 Las entrevistas fueron codificadas de la siguiente manera: Rector (E.1), representante del CGP (E.2) y representante del CAIN (E.3). Las entrevistas se citarán señalando el código, dos puntos y el número de página (ej.: E.1: 1).
} 


\subsection{DISEÑO DE ANÁLISIS}

La información ha sido sometida, luego, a un diseño de análisis crítico del discurso (ACD) especificado en un enfoque histórico, metodología que se vincula más precisamente con un paradigma investigativo crítico (Wodak y Meyer, 2003). El ACD, al relevar el carácter y función ideológico-política del discurso, nos permitirá comprender las tensiones ideológicas, la dinámica de las relaciones de poder y cómo ellas se expresan en el discurso (diálogo y deliberación) curricular desplegado al interior del CEIN, comprendiéndolo como manifestación de un proceso histórico de transformación que afecta a todos los niveles del sistema (especialmente desde 2011), vinculando el contexto nacional con el contexto institucional. Dicho contexto y proceso de transformación produce y se produce en el discurso curricular desplegado en el CEIN. Por ello es que se considera adecuado un enfoque histórico del discurso como especificación del ACD, el cual según Wodak (2003).

(...) trata de integrar la gran cantidad de conocimiento disponible sobre las fuentes históricas con el trasfondo de los ámbitos social y político en los que se insertan los "acontecimientos" discursivos. Además, analiza la dimensión histórica de las acciones discursivas, procediendo a explorar los modos en que los particulares tipos de discurso se ven sujetos a un cambio diacrónico (Wodak y Meyer, 2003: 104).

El diseño de análisis se realizó atendiendo al marco metodológico del $\mathrm{ACD}^{13}$ y considerando a priori cuatro etapas: 1. Elaboración de una matriz de análisis documental, a utilizarse en actas y entrevistas transcritas, 2. Vaciado de información pertinente en la matriz de actas y de entrevistas, 3. Análisis por categoría conceptual de actas y entrevistas por separado y 4 . Análisis por dimensión, sintetizando el análisis de actas y entrevistas. Una primera etapa de análisis consiste en la elaboración de una matriz de análisis documental que vincula los objetivos específicos con una dimensión de análisis, de la cual se desglosan categorías conceptuales apoyadas con un descriptor. A partir de lo anterior se desprende una pregunta orientadora, cuyas respuestas se incluyen en la columna de análisis, tal como lo ilustra el cuadro número 2 :

Cuadro 2. Matriz de análisis documental

\begin{tabular}{lllll}
\hline \multicolumn{1}{c}{ Dimensión } & Categoría conceptual & \multicolumn{1}{c}{ Descriptor } & Pregunta orientadora & Análisis \\
\hline \multirow{2}{*}{ Contex to } & & Incidencia de la & ¿De qué manera incide & \\
histórico/po- & Contexto nacional & presión social en & la presión social en la & \\
lítico & $(2011 / 2014)$ & la reestructura- & reestructuración insti- & \\
& & ción institucional. & tucional? \\
\hline
\end{tabular}

13 En relación al marco metodológico del ACD, desde un enfoque histórico sostiene Wodak: "Tanto la teoría como la metodología son eclécticas, esto es, se incorporan las teorías y los métodos que resultan útiles para comprender y explicar el objeto sometido a investigación" (Wodak y Meyer, 2003: 109). 
La matriz de análisis documental utilizada finalmente en el análisis de actas y entrevistas, acoge los comentarios formales y de contenido señalados por dos expertos investigadores, a quienes recurrimos como validadores del instrumento.

Un primer nivel de análisis consiste en seleccionar y vaciar, en la columna de análisis, información textual (oraciones, párrafos, etc.) de actas y entrevistas, bajo un criterio de pertinencia en relación a la pregunta orientadora. Tras ello se selecciona e incluye citas, correspondientes a nuestros referentes teóricos, bajo el mismo criterio de pertinencia. Además, en esta etapa se integra comentarios preliminares de parte del indagador. A partir de la información seleccionada y de los comentarios preliminares, en un segundo nivel de análisis se intenta sintetizar una respuesta a la pregunta orientadora (desglosada de la categoría conceptual), articulando el discurso de las actas y de las entrevistas, por separado, con el de nuestros referentes teóricos. Un tercer nivel de análisis integra las respuestas por categoría conceptual de las actas y de las entrevistas en un análisis por cada una de las tres dimensiones. En el siguiente cuadro se esquematiza la relación entre las dimensiones y las categorías conceptuales.

Cuadro 3. Dimensiones y categorías conceptuales

\begin{tabular}{ll}
\hline \multicolumn{1}{c}{ Dimensión } & \multicolumn{1}{c}{ Categorías conceptuales } \\
\hline \multirow{3}{*}{ Contexto histórico/político } & Contexto nacional (2011/2014). \\
& Contexto institucional (2011/2014). \\
& Relaciones de poder. \\
& El CEIN. \\
Intereses o ideologías curriculares & Intereses o ideologías curriculares en tensión \\
& Discurso curricular (formas, contenidos, símbolos deliberati- \\
& vos). \\
Deliberación curricular & Discursos curriculares autoritarios v/s discursos deliberativos. \\
& El curriculum construido en el CEIN desde una perspectiva de \\
& deliberación curricular. \\
\hline
\end{tabular}

La confiabilidad de esta indagación se garantiza resguardando principalmente el valor de credibilidad o validez interna (Sandín, 2000), procurando una óptima y saturada recogida de información, junto con la aplicación rigurosa de los métodos de análisis que se han descrito. Se considera que los criterios de transferibilidad (o validez externa) y confirmabilidad u objetividad (Sandín, 2000) no pueden garantizarse en el presente estudio, puesto que, primero, no es su propósito principal relacionar el caso con la generalidad y, segundo, se reconoce y releva la subjetividad del indagador, la cual desde un paradigma investigativo crítico y un carácter cualitativo de la investigación no podría orientarse por criterios de objetividad, no obstante apoyarse en referentes teórico científicos. 
Estudios Pedagógicos XLII, N² 2: 207-226, 2016

EL CONSEJO ESCOLAR DEL INSTITUTO NACIONAL (JOSÉ MIGUEL CARRERA) DESDE UNA PERSPECTIVA DE DELIBERACIÓN CURRICULAR

\section{RESULTADOS OBTENIDOS}

\subsection{CONTEXTO HISTÓRICO POLÍTICO}

El contexto histórico político que enmarca el proceso de transformaciones que se vinculan con una primera etapa del CEIN dotado de carácter resolutivo, se delimitará entre los años 2011 y 2014 y se ha construido asumiendo que existe una "relación dialéctica entre las prácticas discursivas particulares y los ámbitos de acción específicos (...) en que se hallan ubicados" (Wodak, 2003: 104).

Se considera que el año 2011 es referencial, tanto a nivel nacional como a nivel institucional, ello en razón de las tensiones desencadenadas por las movilizaciones estudiantiles de ese año, posibles de estimar como determinantes de la implementación de la política de consejos escolares resolutivos emprendida por la Municipalidad de Santiago dos años más tarde. En tanto, el año 2014 ya corresponde a un periodo de cierta estabilidad en el funcionamiento del CEIN resolutivo, coincidente, además, con el término de la fase de recogida de información para esta indagación. Para efectos de la construcción de este contexto se ha recurrido principalmente a la información entregada por los tres consejeros del CEIN mediante entrevista, siendo fuentes secundarias las actas del Consejo y otros documentos (declaraciones públicas, artículos en prensa, sondeos de opinión, etc.).

Los tres consejeros entrevistados coinciden en considerar el año 2011 como referencial, sosteniendo el consejero directivo que corresponde a aquella "fecha en que se verifica el conjunto de movilizaciones más extensa que ha tenido este país" (E.1:1). Por su parte, el consejero docente señala que "el 2011 es sin lugar a dudas el movimiento histórico educacional más relevante de nuestro país (...), por la gesta, por las características de su movimiento, por la masividad y por el respaldo amplio ciudadano" (E.2: 1), dando cuenta ambas referencias de una percepción que le atribuye gran relevancia a ese hito en términos históricos y políticos ${ }^{14}$.

En cuanto a sus determinantes, los consejeros entrevistados coinciden en vincular los sucesos de 2011 con una percepción de insuficiencia o status quo respecto de los compromisos, acuerdos y reformas emprendidas desde 2006, expresada especialmente por sectores de estudiantes secundarios. A este respecto el consejero estudiantil entrevistado señala:

Hay que partir de la premisa que el escenario previo al 2011 fue el 2006 donde se desencadenó una eventual reforma a la LGE y que hoy por hoy la vemos como la más deficiente y dentro de todo la más significativa de los últimos años. A fin de cuentas fue una reforma con maquillaje y todos sabemos a qué se refiere (E.3: 1).

En tanto, el consejero docente sostiene que: "el estudiantado no ha estado satisfecho desde los inicios de estos movimientos a comienzos de la década del 2000" (E.2: 1).

La presión ejercida por los estudiantes, vinculada con una capacidad relativa o disminuida temporalmente para contrarrestar al movimiento por parte del gobierno de entonces, obliga a este último a un cambio de ministro en la cartera de educación y a la implementación de un plan contingente (no programático) de negociación, concesiones y reformas ${ }^{15}$.

Para mayores antecedentes sobre la secuencia de las movilizaciones estudiantiles en 2011 se recomienda visitar el sitio http://cesocuchile.wordpress.com/especial-movilizaciones/cronologia-de-las-movilizaciones-2011/

15 El 07 de julio de 2011, el entonces Presidente Sebastián Piñera responde al movimiento y a la ciudadanía por cadena 
Resulta relevante, en este contexto, orientándose a indagar en las motivaciones de la política de consejos escolares resolutivos, ilustrar la percepción que los tres consejeros entrevistados tienen del rol desempeñado por los estudiantes secundarios durante el periodo. Sostiene a este respecto el consejero directivo: “(...) a partir de ese momento se sintió un actor estudiantil en la educación media muy fuerte, muy potente, muy consistente, muy sólido y que asumía un rol de protagonismo que antes habían tenido los estudiantes universitarios" (E.1: 1), agregando más adelante que "(...) hubo una gran muestra de fuerza y de ingenio por parte de los grupos que lideraban estas movilizaciones" (E.1: 1). En tanto, el consejero docente considera que el estudiantado secundario "se ve mucho más reactivo, se ve más incisivo si se quiere decir, algo como una especie de estar constantemente pendiente de sus demandas y de que estas lleguen a buen término" (E.2: 1). Estas referencias nos permiten considerar que el estudiantado secundario se posiciona definitivamente como fuerza de presión, incidente en la toma de decisiones políticas y cuya inclusión es vista como necesaria, en términos de maniobra política, tanto por el gobierno central, como por los gobiernos locales (comunales).

Es así como, en 2013, la nueva administración de la comuna de Santiago, encabezada por Carolina Tohá, establece en el marco de su "Planificación y participación ciudadana" aquello que denomina "estrategias participativas", en las cuales se incluye promover la realización de claustros por estamentos, por establecimiento y a nivel comunal y la dotación de carácter resolutivo para los consejos escolares dependientes de la DEM en tres de las seis materias que establece la ley 19.979, ello argumentando que “(...) la única manera de reconstruir las confianzas es escuchando, poniéndose de acuerdo, que es lo que corresponde en una instancia que es educativa" (lanacion.cl, 2013). Es posible vincular la propia elección de Tohá, tras sucesivos mandatos de alcaldes pertenecientes a la coalición de derecha (Lavín, Alcaíno y Zalaquett), y su estrategia de generar espacios de participación, con el contexto de presión social expuesto anteriormente.

El Instituto Nacional asume la estrategia municipal, lo cual le presiona a generar condiciones para la realización e institucionalización de un claustro (realizado durante 2013), junto con institucionalizar el Consejo escolar, organismo que aparece, de pronto y a priori, como un cuerpo colegiado, permanente, con facultades específicas y resolutivas que coinciden con facultades de gobierno institucional. El consejero estudiantil reflexiona de esta forma en relación a la estrategia municipal:

¿Por qué la municipalidad crea este espacio de carácter resolutivo en el consejo escolar?, puedo
tener diferentes postulados, pero lo que sí creo que es importante es, y quizás soy ingenuo o iluso
al pensar, de que quieren tener un proceso de mayor participación de las comunidades escolares
y que sean escuchadas, de que la comunidad en su conjunto se empodere de su espacio, se
empodere de tal manera que ellos mismos puedan decidir las políticas internas dentro de la
comunidad escolar (E.3: 2).

En cuanto al contexto interno y paralelamente a los acontecimientos nacionales durante el periodo señalado, sectores del profesorado, el estudiantado en toma y de los apoderados del Instituto Nacional insatisfechos con la gestión en términos de transparencia, participación,

nacional, proponiendo, entre otros, el "gran acuerdo nacional en educación" (GANE), que incluía la creación de un fondo especial para educación, aumento de becas, reducción de tasas para créditos en educación superior, etc. (fuente: http://www. latercera.com/) 
manejo político-administrativo e infraestructura, etc., presionan por la destitución del hasta entonces Rector Jorge Toro, quien presenta su renuncia en junio de 2013, asumiendo como Rector subrogante Fernando Pérez, inmediatamente tras la renuncia de Toro ${ }^{16}$. El consejero docente caracteriza de esta forma el escenario interno durante el periodo: "El colegio vive hace bastantes años una crisis al interior que tiene que ver con las relaciones laborales y las relaciones institucionales" (E.2: 2).

En este contexto es que el CEIN comienza a sesionar informado de su carácter resolutivo (no reglamentado aún), haciéndolo paralelamente a la realización del claustro; resultando relevante, durante esta primera etapa, el vínculo entre la dinámica de desarrollo del CEIN y la del claustro. Este último, junto con ser positivamente valorado por la comunidad como un espacio participativo, democrático y vinculante, posee manifiestas características refundacionales, ello en virtud de las temáticas que enfrenta y que son sometidas a consideración por parte de la comunidad ${ }^{17}$. La dinámica del claustro y sus resoluciones constituyen muchas veces el contenido de las sesiones del CEIN durante este periodo.

\subsection{INTERESES O IDEOLOGÍAS CURRICULARES}

En esta segunda dimensión se profundiza en los intereses o ideologías curriculares que manifiestan principalmente los representantes del CEIN, ello con el propósito de visibilizar las tensiones ideológicas y las relaciones de poder que se despliegan al interior del CEIN en torno a la construcción curricular, y considerando que "para el ACD la ideología representa un importante aspecto del establecimiento y conservación de unas relaciones desiguales de poder" (Wodak, 2003: 30). En el contexto antes descrito, tales intereses o ideologías curriculares necesariamente se tensionan y lo hacen en relación a una "crisis institucional" declarada, a una reconfiguración de fuerzas internas y a una tendencia refundacional, debiendo inéditamente someterse a consideración por parte de toda la comunidad, tanto en jornadas de claustro, como en consejo escolar. Para efectos de la presentación de resultados en esta dimensión, hemos recurrido como fuente principal a las actas del CEIN y a las entrevistas realizadas a tres de sus consejeros, información que hemos integrado con juicios y categorías de nuestros referentes teóricos.

Durante el periodo resulta manifiesta en las actas del CEIN la inclinación de los consejeros docentes, respaldados por los consejeros directivos, en cuanto a que el consejo general de profesores (CGP) mantenga facultades resolutivas excluyentes en torno a la construcción de curriculum formal, concretado específicamente en los planes y programas de estudios ${ }^{18}$ que en este caso son elaborados por el propio establecimiento. En este sentido señala el consejero directivo entrevistado: “(...) los profesores en este colegio son particularmente quisquillosos respecto de aceptar que gente que no es profesional de la educación tenga incidencia en trabajos técnico pedagógicos" (E.1: 5), mientras que el consejero docente sostiene que: "Sobre el aspecto curricular, el acuerdo como estamento docente es que debe pasar por el CGP” (E.2: 4).

16 Como antecedente, y tras las tensiones internas tras el hito de 2006, la comunidad presiona por la salida del antecesor de Toro, el Rector Omar Letelier.

17 Mayores antecedentes en el sitio web institucional http://institutonacional.cl/, link "claustro".

18 Consideraremos los planes y programas de estudio como aquel texto en donde se establece los objetivos, la selección y secuencia de contenidos y el número de horas por asignatura y nivel. 
Es relevante considerar que durante un primer momento el CEIN se concentra en dotarse de un reglamento, lo cual le presiona a delimitar su ámbito de competencia. Al momento de interpretar la ley 19.979 y la ordenanza 294 en aquello relativo al ámbito curricular, los consejeros docentes y directivos, en ausencia de consejeros estudiantiles, se inclinaron a plantear que la normativa no permite a los consejos escolares resolver sobre aspectos "técnico pedagógicos" (Acta 12/09: 8). Dicha inclinación se sustenta, aparentemente, en aquello prescrito en el párrafo final del artículo 8 de la ley 19.979 en donde se plantea que: "El Consejo no podrá intervenir en funciones que sean de competencia de otros organismos del establecimiento educacional". En tal sentido el curriculum formal, cristalizado particularmente en los planes y programas de estudios, junto con ser identificado por los docentes, directivos y DEM como una materia técnico pedagógica, es visto además como una función para la cual es excluyentemente competente el CGP.

Esta noción nos permite vincular el discurso curricular de tales consejeros con un interés técnico, en términos de Grundy, puesto que subyace allí el propósito de establecer que la construcción de curriculum formal corresponde a una cuestión técnica, distinta de una "construcción cultural", y que existe un grupo de técnicos del curriculum que definen "objetivos pre especificados que determinarán el diseño de la experiencia de aprendizaje", cuya "selección y organización (...) dependerán de la habilidad de los creadores del curriculum" (Grundy, 1991: 50). Es posible además vincular tal noción del curriculum con aquello que T. da Silva denomina teorías curriculares tradicionales, las cuales “(...) al aceptar más fácilmente el status quo, los conocimientos y los saberes dominantes; terminan por concentrarse en cuestiones técnicas" (da Silva, 1999: 6). Sin embargo, y junto con una consideración técnica del curriculum, lo que se desvela de dicho discurso es el interés de resguardo, por parte del cuerpo docente, de la construcción curricular como un "espacio de poder" apropiado (da Silva, 1999: 35).

No obstante el interés técnico subyacente en la consideración del curriculum formal y el resguardo de dicho espacio de poder por parte del cuerpo docente, se manifiesta de parte de los propios consejeros directivos, docentes y del DEM la expectativa de que, mediante pronunciamiento de "la autoridad competente", se otorgue facultad resolutiva en tales materias (Acta 12/09: 9). Dicha idea, manifiesta en acta, es coherente con los intereses curriculares manifestados por los tres consejeros en entrevista, resultando ilustrativa, a este respecto, la reflexión del consejero directivo, quién plantea: "Yo creo, lo he dicho públicamente y lo reitero, que no se requiere ser profesor para poder entregar alguna visión técnica pedagógica o algún concepto que tenga que ver con el sentido común de la educación" (E.1: 5), mientras que el consejero docente sostiene: "El colegio tiene ciertas características que lo hacen tener una visión bastante decimonónica en cuanto a toma de decisiones curriculares. En lo personal yo creo que debiesen abrirse más espacios" (E.2: 5). El consejero estudiantil ratifica lo anterior, planteando:

(...) que lo más importante es que se genere un espacio o una plataforma donde se toque este mismo tema (en referencia al curriculum), pero como tema central, o sea de que UTP, los profesores, los estudiantes, dirección, los paradocentes, todos nos encarguemos de discutir cuáles van a ser las mallas curriculares que nosotros vamos a implantar y de qué forma o qué modelo educativo también (E.3, p.6). 
Si bien de parte de ciertos consejeros se restringe la competencia del CEIN para resolver particularmente sobre planes y programas de estudio, lo que sí se desenvuelve es diálogo en torno a estas y otras materias de relevancia curricular, específicamente en aquellas resueltas durante el claustro 2013. Tales materias, a saber: 1. Visión y misión, 2. Electivos, 3. Plan de idiomas, 4. Reemplazo de la asignatura de Religión y 5. Academias y talleres, son mediadas por el CEIN, espacio en donde se replantean las resoluciones del claustro y se definen estrategias (acciones técnicas, Acta 08/01) y propuestas para su formalización institucional, mas no su modificación, aunque se sostiene, de parte de consejeros estudiantiles, que el CEIN debe "aprobar" tales resoluciones para darles curso institucional (Acta 29/11: 3). Los consejeros despliegan discursos en relación a tales materias en razón de que constituyen resoluciones adoptadas en el claustro 2013, refiriéndose a la "legalidad" de ciertas modificaciones horarias (Acta 12/09: 7), la pertinencia de ampliar la oferta en el caso de idiomas (Acta 12/09: 7), las eventuales consecuencias de que tales resoluciones de claustro colisionen con la competencia del CGP (Acta 12/9: 11), etc.

Tales discursos permiten considerar que existe un interés común de los consejeros en cuanto a promover las resoluciones del claustro, sin embargo en ese diálogo curricular es posible advertir también un interés técnico, puesto que se comprende que las resoluciones del claustro, posibles de caracterizar anticipadamente como un curriculum deliberado, son susceptibles de ser implementadas mediante "acciones técnicas", desvinculadas de la dinámica deliberativa del claustro. En muchos casos el responsable de ejecutar tales acciones técnicas, mandatado consensuadamente por el CEIN, es precisamente la Unidad Técnico Pedagógica (UTP) del establecimiento, organismo que para este efecto cumple el papel de experto o técnico curricular (Acta 6/01: 4, Acta 8/01: 1).

En relación a otras materias curriculares (ranking, jornadas conmemorativas, actos, eventos, etc.), las actas evidencian diálogo y ejercicio de facultad resolutiva por parte de los consejeros (acto conmemorativo 11 de septiembre y actividades fiestas patrias 2013, Acta 12/9: 4-6), decisiones que al centrarse en la gestión de tales actividades no permiten develar intereses ni ideologías curriculares distintas de las relevadas anteriormente.

\subsection{DELIBERACIÓN CURRICULAR}

En esta tercera dimensión se analiza la dinámica del CEIN en cuanto a las condiciones formales en que se manifiesta el diálogo y la eventual deliberación curricular, ello atendiendo a la siguiente referencia de Habermas, quién plantea que: "Todo depende, pues, de las condiciones de la comunicación y de los procedimientos que prestan su fuerza legitimadora a la formación institucionalizada de la opinión y de la voluntad común" (Habermas, 1999: 239). Más precisamente, se centra en la composición del CEIN, vinculándola con las nociones de representatividad, legitimidad y democracia, y en la dinámica formal (condiciones de comunicación y procedimientos) en procesos de diálogo y votación en torno a materias de interés curricular, intentando develar allí si se cumplen condiciones de deliberación en los términos propuestos por Habermas. Las fuentes principales, para efectos de la presentación de estos resultados, son las actas y el reglamento del CEIN ${ }^{19}$, además de las entrevistas realizadas a tres de sus consejeros.

19 Sancionado el 07/11/2013, disponible en http://institutonacional.cl/, link “consejo escolar/reglamento". 
Puede considerarse que la composición del $\operatorname{CEIN}^{20}$ es heterogénea en cuanto a los grados de democracia o respaldo en bases con el que cuentan los distintos consejeros. Los consejeros del CAIN, del CEGPAIN y de los asistentes han sido promovidos al CEIN por sus bases o bien por el mérito de su cargo, adquirido en elecciones universales, mientras que los consejeros directivos y del DEM han sido promovidos únicamente por el mérito de su cargo y por decisión política-administrativa, respectivamente. Ello resulta relevante a la hora de considerar los grados de representatividad con el que, de entrada, cuenta cada uno de los consejeros. Así y todo, ningún estamento ha decidido marginarse de su participación permanente en el CEIN por considerar que tal o cual consejero carece de representatividad o legitimidad, validando con ello el espacio.

Cabe discutir si en presencia de consejeros no electos puede hablarse de una composición del CEIN propicia para la deliberación, ello atendiendo a que en términos de Cohen, citado por Habermas: "La noción de una democracia deliberativa está enraizada en el ideal intuitivo de una asociación democrática en la que la justificación de los términos y condiciones de la asociación tienen lugar a través de la argumentación y del razonamiento público entre ciudadanos iguales" (Cit. en Habermas, 1992: 381). No obstante, resulta relevante la intervención del Rector en sesión del CEIN del 29/11/2013, quién califica a este órgano como "máxima autoridad de la comunidad institutana" (Acta 29/11: 4), agregando en entrevista que: "las grandes decisiones las toma el Consejo escolar y son irrefutables" (E.1: 5), afirmaciones que nos permiten considerar que, superando un heterogéneo grado de representatividad, la condición de "consejero" iguala a los 18 representantes, no existiendo facultad discrecional relevante más que la posibilidad de convocar a sesión extraordinaria por parte del presidente del CEIN (Rector).

En cuanto a la consideración del CEIN como un órgano democrático ${ }^{21}$, resultan relevantes las apreciaciones de los consejeros entrevistados, quienes manifiestan juicios contradictorios al respecto. El consejero directivo cuestiona un carácter plenamente democrático del CEIN señalando que:

(...) a veces ocurre que no llega ni uno solo de un estamento y eso dificulta el funcionamiento, altera la dinámica, pero me atrevo a asegurar que cuando el consejo va adquiriendo cierta frecuencia en su funcionamiento, ciertas decisiones que impactan mediana o poderosamente en el andar del colegio, es una buena estructura organizativa (E.1: 4).

En tanto, el consejero docente sostiene que: "se puede considerar una instancia democrática, pero requiere de una mayor participación” (E.2: 3). Por su parte, y cuestionando más profundamente la dinámica democrática interna del CEIN, el consejero estudiantil señala que: "La gente que participa del Consejo escolar no comprende que ellos no son los que deben tomar las decisiones, sino que ellos van a representar las decisiones de los estamentos, y ese es un problema" (E.3: 5).

20 La composición del CEIN conforme al reglamento, es la siguiente: 4 estudiantes, 3 docentes, 3 apoderados, 3 asistentes de la educación, 1 directivo docente, 2 directivos y 2 representantes de la DEM.

${ }_{21}$ Habermas, citando a Dahl, reconoce las siguientes condiciones en un proceso democrático: a. inclusión de todos los afectados, b. oportunidades igualmente distribuidas e igualmente eficaces de participación en el proceso político, c. igual derecho a voto en las decisiones, d. el mismo derecho a la elección de temas y en general al control del orden del día, e. información suficiente y buenas razones que permitan formar una comprensión articulada de la materia necesitada y de los intereses en conflicto (Cit. en Habermas, 1998: 394). 
La dinámica de sesión, manifiesta en las actas, es recurrente. Por lo general, el Rector, que además por reglamento preside CEIN, es quién plantea la tabla, esta última, por lo común, esbozada al finalizar la sesión anterior. Luego ofrece la posibilidad para que los restantes consejeros formulen observaciones y/o nuevos puntos a la tabla. Los turnos de habla para la profundización de los puntos se consiguen levantando la mano y tras la venia del presidente del consejo. No se verifica en las actas límites a los turnos de habla, salvo aquellos que corresponden a interrupciones o contra argumentaciones en caso de debate. En tanto, cuando un determinado punto se considera resuelto (saturado) o en consenso, es el presidente, aunque no de manera excluyente, quién propone dar paso al punto siguiente. En tal sentido, no se advierten rasgos manifiestos de sesgo, discriminación, censura u otro, más que aquellos que pueden derivarse de asimétricos grados de información, niveles de expertise, manejo retórico, etc.

Menos común es la dinámica de votaciones al interior del consejo. A este respecto cabe destacar la sesión del 05 de septiembre de 2013, instancia en la cual el CEIN sometió a votación, tras consideración y debate, el dar curso o descartar proyectos presentados por la comunidad, muchos de los cuales poseen relevancia curricular (Gestión técnico pedagógica, Mejoramiento de la eficiencia en Física y Academia conjunto folclórico). La dinámica de votaciones es, en general, la siguiente: se presentan depuradas las alternativas divergentes y luego se solicita levantar la mano en favor o en contra de cada una de ellas. La transcripción de las actas no considera, en estos casos, el detalle de las votaciones, lo cual hubiese permitido advertir alineaciones, tensiones, etc. Así y todo existen puntos sometidos a votación que cuentan, sino con una unanimidad, con una amplia mayoría al interior del CEIN. En tanto, aquellas votaciones más cerradas permiten considerar la posibilidad de divergencia y ausencia de presiones y coerción.

\section{CONCLUSIONES Y DISCUSIÓN}

A partir del análisis anterior, en sus tres dimensiones y contrastando la dinámica del CEIN con las condiciones de deliberación propuestas por Cohen y corroboradas por Habermas (1998), es posible concluir que:

a. El curriculum construido en el CEIN se sostiene en un intercambio regulado de informaciones y razones, ello atendiendo a que el CEIN es un espacio reglamentado en donde "se presentan propuestas y se someten a crítica" (Habermas, 1998: 382).

b. Las razones que allí se vierten son inclusivas en el sentido de que, en principio, en el CEIN se representa a la totalidad de los estamentos. No obstante es facultad discrecional del CEIN incluir o excluir a otros agentes "afectados por las resoluciones" (Habermas, 1998: 382), distintos de los 18 consejeros. Tales razones son además públicas, lo cual se confirma, entre otros, con la publicación de actas.

c. No se advierten coerciones internas ni externas manifiestas durante el proceso mismo de deliberación curricular al interior del CEIN.

d. Las deliberaciones curriculares al interior del CEIN se orientan a "alcanzar un acuerdo racionalmente motivado y pueden proseguirse ilimitadamente o retomarse en cualquier momento" (Habermas, 1998: 382) y eventualmente se les pone término mediante un acuerdo mayoritario, establecido a través de consenso o mediante votación. 
e. No obstante encontrarse limitado en cuanto a la resolución de materias técnico-pedagógicas (identificadas con los planes y programas de estudio), las deliberaciones al interior del CEIN se extienden, en principio, a la totalidad de las materias ${ }^{22}$.

f. Las deliberaciones curriculares al interior del CEIN, dadas en un contexto de crisis institucional, de reconfiguración de fuerzas internas y de tendencia refundacional, necesariamente se extienden a "la interpretación de las necesidades y al cambio de actitudes y preferencias prepolíticas” (Habermas, 1998: 382-383).

Planteado de tal forma, es posible concluir que el CEIN se constituye efectivamente en un espacio de deliberación curricular. Sin embargo, queda aún por discutir una serie de implicancias que tensionan dicha afirmación.

Ante todo, cabe discutir si el CEIN resolutivo, constituido como un espacio de deliberación (en general), se ve menoscabado como tal en virtud de que responde originariamente a intereses políticos, especificados en una estrategia, desplegados desde el municipio. Dicha implicancia permite aún suponer que, no obstante constituirse en un espacio de deliberación, el CEIN resolutivo responde al propósito de orientar “desde arriba", especialmente al estudiantado del Instituto Nacional, hacia una vía institucional de establecimiento de diálogo y acuerdo, ello considerando su rol en contextos nacionales y locales de presión. De la misma forma, y atendiendo a un contexto de crisis interna muy bien ilustrada por la renuncia forzada y sucesiva de dos rectores, el CEIN resolutivo aparece nuevamente como un dispositivo dispuesto "desde arriba", orientado, esta vez, a reconducir las fuerzas de ciertos sectores de la comunidad con un propósito de estabilidad interna.

Por su parte, es posible discutir aún de qué manera el interés manifestado por el estamento docente, en cuanto a restringir la resolución de temas técnico-pedagógicos por parte del CEIN, lo afecta como espacio de deliberación curricular, ello atendiendo a la condición de que "las deliberaciones se deben extender, en principio, a la totalidad de las materias". Si bien el CEIN revela una dinámica deliberativa en materias de interés curricular (razonamiento inclusivo, acuerdo mayoritario, etc.), esta se encuentra limitada por el hecho de no poder someter a votación vinculante para la comunidad cuestiones relativas a los planes de estudio. Ello permite suponer que el CEIN se ha omitido de dialogar y/o deliberar acerca de los planes de estudio, lo cual no implica solamente votación vinculante para la comunidad, porque se han restringido sus facultades resolutivas en esta materia.

Este punto se vincula con otra discusión, relacionada esta vez con la importancia de relevar aquellos procesos informales de formación de la opinión en el espacio públicopolítico, ello atendiendo a la crítica planteada por Habermas al modelo de democracia deliberativa propuesto por Cohen. Una auto comprensión del CEIN como espacio de deliberación curricular y su desenvolvimiento práctico como tal, está influida por procesos informales de formación de la opinión, caracterizados por tensiones y consensos dados a nivel de agentes, grupos de interés, departamentos de asignatura, colectivos políticos, gremios, estamentos u otras instituciones internas y/o externas en el ámbito curricular, comprendiéndolo como un espacio de poder.

Es posible también abrir una discusión en torno a la calidad de la democracia al interior del CEIN, ello considerando los cuestionamientos realizados por los consejeros directivo y docente respecto de la participación real de los estamentos, punto que puede vincularse con

Al respecto sostiene el consejero directivo: "no hay temas vedados para el Consejo escolar" (E.1, p.2). 
un desarrollo aún prematuro del CEIN y con el hecho de que se perciba de parte de ciertos agentes como una política "desde arriba", aspectos ambos que se relacionarían con una débil apropiación (empoderamiento) del espacio por parte, especialmente, del estudiantado. Cabe plantear aquí también la crítica formulada por el consejero estudiantil en cuanto a que en el CEIN "se plantean juicios personales y no juicios de base", lo cual abre una relevante discusión en cuanto a los principios democráticos que inspiran las prácticas organizativas de los distintos estamentos (democracia directa, democracia representativa, mixto, etc.), principios y formas que repercuten necesariamente en el CEIN.

\section{REFERENCIAS BIBLIOGRÁFICAS}

Apple, M., \& Beane, J. (2005). Escuelas democráticas. Madrid: Ediciones Morata.

Albert, M. (2007). La investigación educativa: claves teóricas. Madrid: McGraw-Hill.

Da Silva, T. (1999). Documentos de identidad: una introducción a las teorías del currículo. Belo Horizonte: Autentica.

DEM. (2014). Consejos escolares. Recuperado desde http://santiago.k12.cl/icore/viewcore/161446

Ferrada, D. (2001). Comunidades de entendimiento: una propuesta educativa desde la perspectiva crítica / comunicativa del curriculum. Pensamiento Educativo, 29, 297-317.

Ferrada, D. (2008). Enlazando mundos: un modelo pedagógico que construye esperanzas de igualdad e inclusión en escuelas públicas. Revista de Estudios y Experiencias en Educación, 14, 37-52.

Giroux, H. (1983). Teorías de la reproducción y la resistencia en la nueva sociología de la educación: un análisis crítico. Recuperado desde: http://www.pedagogica.edu.co/storage/rce/ articulos/17_07pole.pdf

Grundy, S. (1998). Producto o praxis del curriculum. Madrid: Ediciones Morata.

Habermas, J. (1987). La Acción Comunicativa. Madrid: Editorial Taurus.

Habermas, J. (1982). Conocimiento e Interés. Madrid: Editorial Taurus.

Habermas, J. (1998). Facticidad y Validez. Madrid: Trotta.

Habermas, J. (1999). Tres modelos normativos de democracia. En La inclusión del otro: estudios de teoría política (pp. xx-xx). España: Paidos Ibérica.

Habermas, J. (1999). El vínculo interno entre estado de derecho y democracia. En La inclusión del otro: estudios de teoría política (pp. xx-xx). España: Paidos Ibérica.

Morra, L., \& Friedlander, A. (2001). Evaluaciones mediante Estudios de Caso. Washington D.C.: OED.

Ruiz, J. (1996). Metodología de la investigación cualitativa. Bilbao: Universidad de Deusto.

Sandín, M. (2000). Criterios de validez en la investigación cualitativa: de la objetividad a la solidaridad. Revista de Investigación Educativa, 18(1), 223-242.

Stake, R. (1998). Investigación con estudios de casos. Madrid: Ediciones Morata.

Wodak, R., \& Meyer, M. (2003). Métodos de análisis crítico del discurso. Barcelona: Gedisa.

Wodak, R. (2003). El enfoque histórico del discurso. En Métodos de análisis crítico del discurso, 101-142, Barcelona: Gedisa. 